\title{
Hierarchical approach to aggregate equilibria
}

\author{
Karsten Vogtt $\odot,{ }^{*}$ Gregory Beaucage $\odot,{ }^{\dagger}$ Kabir Rishi $\odot$, Hanqiu Jiang, and Andrew Mulderig \\ Chemical and Materials Engineering, University of Cincinnati, Cincinnati, Ohio 45221, USA
}

(Received 19 July 2019; revised manuscript received 28 September 2019; published 6 November 2019)

\begin{abstract}
Hierarchical aggregation is generally viewed as a kinetic phenomenon governed by kinetic growth laws, such as in the Smoluchowski equation, and modeled using diffusion or reaction limited kinetic growth models. Some aggregates, especially those controlled by surface grafting or surfactants, display reversible stability. For these equilibrated aggregates a simple thermodynamic model is proposed to describe the size distribution and the enthalpy and entropy of aggregation. The model uses the average degree of aggregation, $z_{i(i-1)}$, as the central quantifying parameter. Here $i$ is an index reflecting the hierarchical level of structure in an aggregate, for instance, composed of crystals $(i=0)$, clustered primary particles $(i=1)$, aggregates $(i=2)$, and agglomerates of aggregates $(i=3)$. A change in Gibbs free energy for aggregation is given by $\Delta G_{i(i-1)}=-R T \ln \left(1 / z_{i(i-1)}\right)$ for each level $(i>0)$. This expression is advantageous since the degree of aggregation is directly determined in smallangle neutron and x-ray scattering, by transmission electron microscopy, simulation, or through spectroscopy. The atomistic hierarchical model enables an understanding of the mechanism of equilibrium aggregation since it provides expressions for entropy and enthalpy of aggregation at each structural/thermodynamic level. The model can be extended to describe pseudoequilibrium for industrially relevant materials such as condensation polymers. Applications in organic pigments and wormlike micelles are also briefly demonstrated.
\end{abstract}

DOI: 10.1103/PhysRevResearch.1.033081

\section{INTRODUCTION}

Aggregation phenomena are widely experienced. Aggregates are of industrial importance as high surface area materials such as carbon black and silica. Aggregates can also provide unique optical and mechanical properties utilized in pigments and reinforcing agents. Most aggregates are kinetically formed, locking in an unstable, high surface area structure through rapid, often high temperature and/or concentration processes through various rapid quench operations [1-5]. Some aggregates are self-assembled in a thermodynamically stable state, such as micellar aggregates, surfactant treated organic pigments, and even molecular aggregates in reversible polymerization reactions such as in the synthesis of polydimethyl siloxane, polyesters, and polyamides. Further, some aggregations of kinetic origin might be modeled using a pseudothermodynamic approach where accumulated strain mimics thermal motion [6-8].

For equilibrated aggregation, micellization, or reversible polymerization, a distribution of products with a plethora of reaction pathways exists. Formation of an aggregate with a given number of subunits can be achieved by a number of routes involving various aggregation or dissociation pathways. Because of this the conventional law of mass action

\footnotetext{
*karsten.vogtt@uni-dortmund.de

$\dagger$ gbeaucage@gmail.com

Published by the American Physical Society under the terms of the Creative Commons Attribution 4.0 International license. Further distribution of this work must maintain attribution to the author(s) and the published article's title, journal citation, and DOI.
}

is not particularly useful for equilibrium aggregation or selfassembly.

Chemical equilibria are usually quantified by postulating a chemical potential $\mu_{i}$ that drives the conversion of the given chemical species $i$. It is defined as the partial derivative of the free energy $G_{i}$ with the change of molar particle number $n_{i}$ and can be expressed via a reference potential $\mu_{i}^{\circ}$ and the activity $a_{i}$ of the particles of type $i$. Commonly,

$$
\mu_{i}(\Pi, T)=\partial G_{i}(\Pi, T) / \partial n_{i}=\mu_{i}^{\circ}(\Pi, T)+R T \ln a_{i} .
$$

Under dilute conditions or assuming ideal behavior, $a_{i}$ can be replaced by the mole fraction $x_{i}$. At constant temperature and pressure the change $\Delta G$ of the molar Gibbs free energy for the reaction as compared to a standard free energy $\Delta G^{\circ}=\sum \nu_{i} \mu_{i}^{\circ}$ is given by

$$
\Delta G=\sum v_{i} \mu_{i}=\Delta G^{\circ}+R T \sum v_{i} \ln a_{i},
$$

where $v_{i}$ denotes the stoichiometric coefficient. For a simple multistage chemical reaction $i=\{0,1,2, \ldots\}$, the reaction scheme may be written as

$$
v_{0} M_{0} \stackrel{K_{1, c}}{\leftrightarrow} v_{1} M_{1} \stackrel{K_{2, c}}{\leftrightarrow} v_{2} M_{2} \stackrel{K_{3, c}}{\leftrightarrow} \cdots .
$$

Here, $M_{i}$ denotes the chemical species. At equilibrium, $\Delta G=0$ and Eq. (2) can be employed to define the standard Gibbs free energy $\Delta G^{\circ}$ via the equilibrium constant $K_{c}^{\circ}$ according to the law of mass action,

$$
\begin{aligned}
\frac{\Delta G^{\circ}}{R T} & =-\ln \left\{\frac{\left(c_{1}\right)^{\nu_{1}}}{\left(c_{0}\right)^{\nu_{0}}} \frac{\left(c_{2}\right)^{\nu_{2}}}{\left(c_{1}\right)^{\nu_{1}}} \cdots\right\} \\
& =-\ln \left(\prod_{i} K_{i, c}\right)=-\ln \left(K_{c}^{0}\right),
\end{aligned}
$$




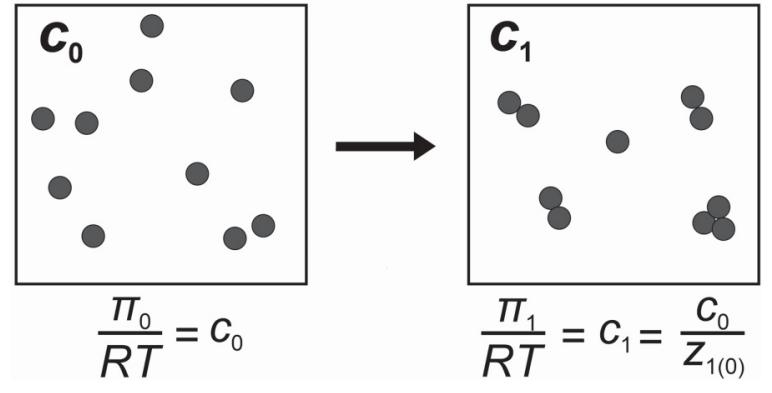

FIG. 1. The decrease of the osmotic pressure $\Pi_{0}$ upon aggregation or bond formation to $\Pi_{1}$ can be expressed as $c_{1}=\frac{c_{0}}{z_{1(0)}}$ via the concentration $c_{0}$ in the monomeric state and the average aggregation number $z_{1(0)}$. The aggregated state can be perceived as a higher structural level $i=1$ assembled from monomeric elementals $i=0$ yielding the size distribution $N_{1}\left(n_{0}\right)$.

where $c_{i}$ is the molar concentration and $K_{c}^{0}$ the equilibrium constant of the overall reaction. This approach is well tried and yields concise expressions for chemical reactions with discrete stoichiometric coefficients. However, for reactions, such as aggregation, micellization, or polymerization, which lead to a distribution of products, even the setup of a reaction scheme as given in Eq. (3) can be problematic. For free, nonspecific aggregation a plethora of reaction paths exist that lead to the formation of an aggregate with a given number of subunits. It may be formed by the association of smaller aggregates or by the dissociation of subunits from larger aggregates. In the above approach, each of these steps along the path would need to be accounted for as a distinct equilibrium reaction with an equilibrium constant $K_{i, c}$. Moreover, the standard free energy $\Delta G^{\circ}$ obtained by the approach refers to a reference state. The associated equilibrium constant $K_{c}^{0}$ bears units depending on the stoichiometry of the reaction. This, and the $a$ priori postulation of the chemical potential, render an intuitive assessment of the potential's characteristic enthalpic and entropic contributions difficult and complicates comprehension of the underlying driving forces. Although kinetic models describing both reversible and irreversible aggregation over different reaction pathways have been simulated, an estimation of the thermodynamic free energy change based on the hierarchical structural parameters as explained here has not been explored [9-13].

\section{THEORY}

An equilibrated aggregate system consists of elemental particles that associate and dissociate from each other, but also exhibit a constant observable (osmotic) pressure. The definition of an elemental particle depends on the nature of the system. A thermodynamic hierarchy is defined by the driving force or chemical potential difference that leads to the assembly of that particular level $(i \geqslant 0)$, which differs somewhat from more traditional structural hierarchies.

\section{A. Atomistic model and the osmotic pressure}

Consider a system of $N_{0}^{T}$ elemental particles dispersed in a volume, $V$, Fig. 1 . If the elemental particles remain monomeric and behave ideally, one would expect to measure an (osmotic) pressure

$$
\Pi_{0}=\left(\frac{N_{0}^{T}}{V}\right) R T,
$$

where $c_{0}=\left(\frac{N_{0}^{T}}{V}\right)$ is the total concentration of elemental particles in moles per volume. Upon aggregation, the (osmotic) pressure will decrease from $\Pi_{0}$ to $\Pi_{1}$. This reduction can be expressed via the second virial coefficient $B_{2}$, indicating interactions that lead to clustering. At the same time, one may express the pressure decrease by the average number of elementals per aggregate, $z_{1(0)}=\frac{c_{0}}{c_{1}}$,

$$
\frac{\Pi_{1}}{R T} \approx c_{0}+B_{2}\left(c_{0}\right)^{2}+\cdots=\frac{c_{0}}{z_{1(0)}} .
$$

The subindex " 1 " on $z_{1(0)}$ expresses that the average aggregation number belongs to the first aggregated state, whereas the subindex " 0 " highlights parameters of the monomeric particles. Because the aggregated particles $i=1$ are assembled from monomers $i=0$, their number distribution as a function of subunit number is designated as $N_{1}\left(n_{0}\right)$. Here, $n_{0}$ indicates the number of elemental particles in an aggregate analogous to the degree of polymerization. Note that this distribution contains the free, unbound monomers as $N_{1}\left(n_{0}=1\right)$. The total number of elementals, $N_{0}^{T}$ is given by

$$
N_{0}^{T}=\int_{0}^{\infty} N_{1}\left(n_{0}\right) n_{0} d n_{0},
$$

and the total number of aggregated particles $N_{1}^{T}$ can be defined as

$$
N_{1}^{T}=\int_{0}^{\infty} N_{1}\left(n_{0}\right) d n_{0} .
$$

Defining $v_{0}$ as the volume of an elemental and $N_{\Omega, 0}=\frac{V}{v_{0}}$ as the number of volume elements of size $v_{0}$ within the total volume $V$, the total volume fraction, $\phi$ is given by

$$
\phi=\left(\frac{v_{0}}{V}\right) \int_{0}^{\infty} N_{1}\left(n_{0}\right) n_{0} d n_{0}=\frac{N_{0}^{T}}{N_{\Omega, 0}} .
$$

Considering the transition from the purely monomeric state with the overall concentration $c_{0}$ to the aggregated state $c_{1}$ in Fig. 1, the change in the molar Gibbs free energy, $\Delta G_{1(0)}=\mu_{0}^{\circ}-\mu_{1}$, is given by

$$
\begin{aligned}
\Delta G_{1(0)} & =-R T \ln \left(\frac{N_{1}^{T}}{N_{0}^{T}}\right)=-R T \ln \left(\frac{1}{z_{1(0)}}\right) \\
& =-R T \ln \left(K_{1(0)}\right) .
\end{aligned}
$$

Note that in this form $\Delta G_{1(0)}$ denotes the free energy of dissociation. This is done to allow direct comparison with previous results from the literature (see below).

Equation (10) is valid if the very same driving force assembles all the aggregate types. The formulation makes no assumptions on reaction paths leading to the aggregated particles in the hierarchical level, $i=1$. Further aggregation governed by a different chemical potential of particles at level $i=1$, would constitute a new hierarchical level $i=2$, Fig. 2 . The assembly of aggregate distribution, $N_{2}\left(n_{0}\right)$, in the second 


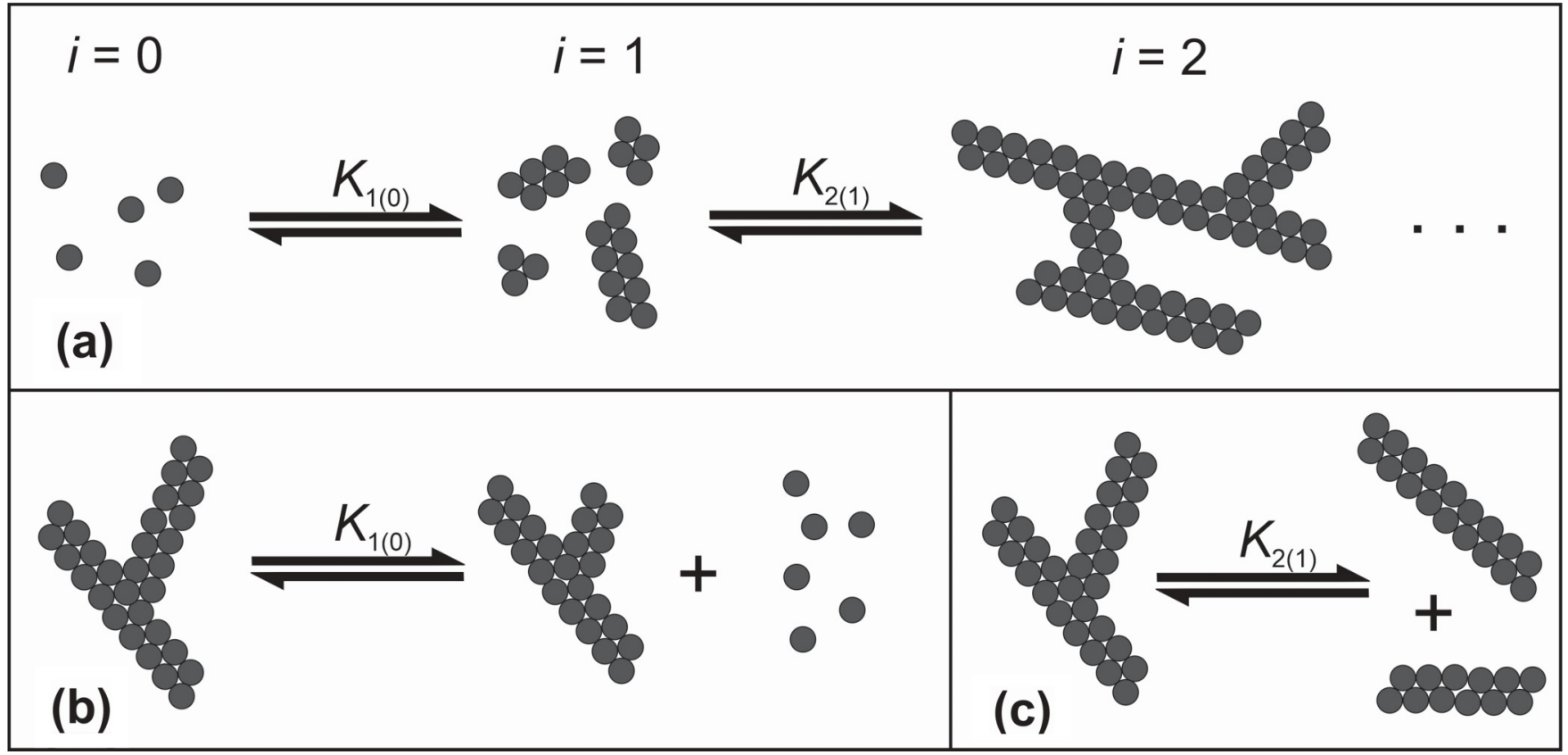

FIG. 2. The hierarchical principle of particle association/dissociation. A level of hierarchy $i$ is defined by the binding motif and its underlying driving force and not by the number of subunits that associate (a). Likewise, the dissociation from large aggregates is categorized according to which kind of bonds are decomposed (b) and (c).

hierarchical level is governed by free energy change, $\Delta G_{2(0)}$. In this case, the (osmotic) pressure in Eq. (6) would further decrease by a factor of $\frac{1}{z_{2(1)}}$, and

$$
\begin{aligned}
\Delta G_{2(0)} & =-R T \ln \left(\frac{N_{2}^{T}}{N_{0}^{T}}\right)=-R T \ln \left(\frac{1}{z_{2(1)} z_{1(0)}}\right) \\
& =-R T \ln \left(K_{2(1)} K_{1(0)}\right) .
\end{aligned}
$$

The hierarchical approach yields formally the same product of equilibrium constants given by the law of mass action but it uniquely links the free energy directly to the average degree of aggregation for each hierarchical level.

Combining Eqs. (6) and (10) one finds that

$$
\frac{\Pi_{1}}{R T}=c_{0} \exp \left(\frac{-\Delta G_{1(0)}}{R T}\right) .
$$

$c_{0}$ is the molar concentration of monomers and using the ideal gas approximation, $c_{0}=\frac{\Pi_{0}}{R T}$. For the logarithm of the ratio of osmotic pressure at two different temperatures $T^{\prime}$ and $T^{\prime \prime}$ one may write

$$
\begin{aligned}
\ln \left[\frac{\Pi_{1}\left(T^{\prime}\right)}{\Pi_{1}\left(T^{\prime \prime}\right)}\right] & =\frac{-\Delta G_{1(0)}}{R T^{\prime}}+\frac{\Delta G_{1(0)}}{R T^{\prime \prime}} \\
& =-\frac{\Delta H_{1(0)}}{R}\left(\frac{1}{T^{\prime}}-\frac{1}{T^{\prime \prime}}\right),
\end{aligned}
$$

which represents the integrated form of the ClausiusClapeyron equation (the usual assumption that the volume of the condensed phase must be ignored is not necessary in this approach since the condensed phase is counted as a vapor particle). This underlines that the hierarchical approach also describes simple phase equilibria.
While the above consideration started with the ideal gas law, nonetheless the existence of a chemical potential $\mu_{i}$ was presupposed in the derivation of the equilibrium constant $K_{1(0)}$. This was done in order to demonstrate that the atomistic hierarchical approach is compatible with the law of mass action and the concept of a chemical potential. However, the hierarchical definition of the free energy $\Delta G_{1(0)}=$ $-R T \ln \left(\frac{1}{z_{100}}\right)$ can likewise be derived using a purely atomistic approach as discussed below.

\section{B. Differential expression for equilibrated population balances}

If one considers a distribution of aggregated particles consisting of two hierarchical levels, monomeric elementals $(i=0)$ and aggregates of them $(i=1)$, that give rise to a stable (osmotic) pressure, then one may state

$$
\left\langle\Psi N_{1}\left(n_{0}\right) d n_{0}+d N_{1}\left(n_{0}\right)\right\rangle_{t}=0 .
$$

This equation shows that averaged over an appropriate time $t$, the change of number of subunits $N_{1}\left(n_{0}\right) d n_{0}$ occurring to the aggregates times a constant $\Psi$ will be compensated by a corresponding change of aggregate number $d N_{1}\left(n_{0}\right)$. It expresses that any number of subunits $d n_{0}$ leaving or joining an aggregate will correspond to a change of free particle number $d N_{1}\left(n_{0}\right)$. Referring to the chemical potential and the Gibbs equilibrium principle, Rusanov derived a very similar relation $[14,15]$. If one considers noncooperative aggregation with a constant $\Psi$ that is not a function of $n_{0}$, one obtains an exponential distribution function $N_{1}\left(n_{0}\right)$ (see below).

Setting $\Psi=\frac{1}{\left.z_{10}\right)}$ yields the probability distribution with the maximum entropy according to information theory (that is related but not identical to the entropy defined via thermodynamics) [16]. One might also apply a constant to the right-hand term of Eq. (14), but then this would describe 
the opposite reaction, i.e., the association reaction. Note that further hierarchical levels would simply add up as continuing summands, $\Psi_{i} N_{i}\left(n_{i-1}\right) n_{i-1} d n_{i-1}+n_{i-1} d N_{i}\left(n_{i-1}\right)$.

Equation (14) represents a first-order differential equation of the form $\Psi f(x) d x+d f(x)=0$ so that $\frac{d N_{1}\left(n_{0}\right)}{d n_{0}}+\frac{N_{1}\left(n_{0}\right)}{z_{1(0)}}=$ 0 . This results in an exponential distribution function

$$
N_{1}\left(n_{0}\right)=C \exp \left(-\frac{n_{0}}{z_{1(0)}}\right)
$$

where $C$ is a constant. From Eqs. (7) and (15), the total number of elementals is given by

$$
N_{0}^{T}=C \int_{0}^{\infty} \exp \left(\frac{-n_{0}}{z_{1(0)}}\right) n_{0} d n_{0} .
$$

Note that the integration limits from $n_{0}=0$ to $n_{0}=\infty$ were considered for the sake of conciseness of the resulting expressions. In the resulting exponential distribution $n_{0}$ exhibits an offset value of -1 that can be neglected if $z_{1(0)}$ is sufficiently large. The definite integral in Eq. (16) can be solved via integration by parts, $\int u d v=u v-\int v d u$, such that $u=n_{0}$ and $d v=\exp \left(\frac{-n_{0}}{z_{1(0)}}\right) d n_{0}$. Then $d u=d n_{0}$ and $v=$ $-z_{1(0)} \exp \left(\frac{-n_{0}}{z_{1(0)}}\right)$, and the total number of elemental particles is

$$
N_{0}^{T}=\left.C\left\{-n_{0} z_{1(0)} \exp \left(\frac{-n_{0}}{z_{1(0)}}\right)-z_{1(0)}{ }^{2} \exp \left(\frac{-n_{0}}{z_{1(0)}}\right)\right\}\right|_{n_{0}=0} ^{n_{0}=\infty} .
$$

For the upper bound, $n_{0}=\infty$, the first term on the right side of Eq. (17) results in the indeterminate form, $\frac{-\infty}{\infty}$. From the L'Hôpital's rule, $\lim _{n_{0} \rightarrow \infty}\left[-n_{0} z_{1(0)} / \exp \left(\frac{-n_{0}}{z_{1(0)}}\right)\right]=0$.

Additionally, the second term at $n_{0}=\infty$ equals 0 . Similarly, for the upper bound, $n_{0}=0$, the first term equals 0 and the second term equals $-z_{1(0)}^{2}$. Thus the constant, $C=\frac{N_{0}^{T}}{z_{1(0)}^{2}}$. The resulting number distribution of aggregated particles from Eq. (15) is

$$
N_{1}\left(n_{0}\right)=\left(\frac{N_{0}^{T}}{z_{1(0)}^{2}}\right) \exp \left(\frac{-n_{0}}{z_{1(0)}}\right) .
$$

Dividing both sides of Eq. (18) by $N_{\Omega, 0}$ and substituting for the the particle volume fraction from Eq. (9) upon rearragnement results in

$$
\frac{-n_{0}}{z_{1(0)}}=\ln \left(\frac{\left(z_{1(0)}\right)^{2}}{\phi}\right)+\ln \left(\frac{N_{1}\left(n_{0}\right)}{N_{\Omega, 0}}\right) .
$$

Combining Eqs. (14) and (19) results in the following differential expression:

$$
\begin{aligned}
& \left\langle N_{1}\left(n_{0}\right)\left\{\ln \left[\frac{\left(z_{1(0)}\right)^{2}}{\phi}\right]+\ln \left[\frac{N_{1}\left(n_{0}\right)}{N_{\Omega, 0}}\right]\right\} d n_{0}-n_{0} d N_{1}\left(n_{0}\right)\right\rangle_{t} \\
& \quad=0 .
\end{aligned}
$$

The term $N_{1}\left(n_{0}\right) \ln \left(\frac{N_{1}\left(n_{0}\right)}{N_{\Omega, 0}}\right)$ can be identified as the mixing entropy. Accordingly, the term $\ln \left[\frac{\left(z_{1(0)}\right)^{2}}{\phi}\right]$ represents the free molar energy of dissociation $\Delta G_{1(0)}^{d}$. The sum of the two logarithmic terms is the chemical potential of the aggregation process. The average number $z_{1(0)}$ in an aggregate is therefore given by

$$
z_{1(0)}=\phi^{1 / 2} \exp \left(\frac{-\Delta G_{1(0)}^{d}}{2 R T}\right) .
$$

Cates obtained the very same expression for wormlike micelles via minimization of the same thermodynamic potential within the mean field/Flory-Huggins solution theory using Lagrange multipliers [17-19]. In Cates' work $\Delta G_{1(0)}^{d}=\Delta G_{1(0)}^{S C}$, an analog to the scission free energy. Here it is shown that the relation in Eq. (21) applies to all systems where aggregation is driven by this kind of potential, i.e., a term describing attractive interaction balanced by the mixing entropy.

The change in chemical potential from the monomeric state to the aggregated state, with the total change in mixing entropy, can be obtained from the indefinite integral of Eq. (19) with respect to $z_{1(0)}$. Substituting for the distribution function, $N_{1}\left(n_{0}\right)$ from Eq. (18) and noting that the term $\int \frac{-n_{0}}{z_{1(0)}} d z_{1(0)}$ on both sides of the expression nullifies, results in

$$
2 \int \ln \left(\frac{z_{1(0)}}{\phi^{1 / 2}}\right) d z_{1(0)}-2 \int \ln \left[\frac{z_{1(0)}\left(N_{\Omega, 0}\right)^{1 / 2}}{\left(N_{0}^{T}\right)^{1 / 2}}\right] d z_{1(0)}=0 .
$$

The two integral terms in Eq. (22) can be solved through integration by parts, $\int u d v=u v-\int v d u$. For the first term let $u=\ln \left(\frac{z_{1(0)}}{\phi^{1 / 2}}\right)$; then $d u=\frac{d z_{1(0)}}{z_{1(0)}}$. Also, let $d v=d z_{1(0)}$; then $v=z_{1(0)}$

Similarly for the second term, let $u=\ln \left[\frac{z_{1(0)}\left(N_{\Omega, 0}\right)^{1 / 2}}{\left(N_{0}^{T}\right)^{1 / 2}}\right]$; then $d u=\frac{d z_{1(0)}}{z_{1(0)}}$. Also, let $d v=d z_{1(0)}$; then $v=z_{1(0)}$, resulting in

$$
\ln \left(\frac{z_{1(0)}^{2}}{\phi}\right)+\ln \left\{\frac{\left(\frac{N_{0}^{T}}{z_{1(0)}}\right)}{N_{\Omega, 0}}\right\}=\ln \left(z_{1(0)}\right) .
$$

To obtain the overall free energy associated with the dissociation process in a hierarchical system both sides of Eq. (23) are multiplied by $R T$. Since $z_{1(0)}=\frac{c_{0}}{c_{1}}=\frac{N_{0}^{T}}{N_{1}^{T}}$, one obtains

$$
\Delta G_{1(0)}=R T \ln \left(z_{1(0)}\right)=R T\left\{\ln \left[\frac{\left(z_{1(0)}\right)^{2}}{\phi}\right]+\ln \left(\frac{N_{1}^{T}}{N_{\Omega, 0}}\right)\right\},
$$

in agreement with Eq. (10) and the derivation based on a chemical potential. The above potential consists of a free association/dissociation energy, $\Delta G_{1(0)}^{d}=R T \ln \left[\frac{\left(z_{1(0)}\right)^{2}}{\phi}\right]$, and a mixing entropy, $\Delta G_{1(0)}^{m}=\ln \left(\frac{N_{1}^{T}}{N_{\Omega, 0}}\right)$. Many reactions, e.g., condensation processes, can be described with this simple model.

More complex reactions can be described depending on the approach for $\Psi$ in Eq. (10). If clustering occurs in a cooperative manner, i.e., the aggregation is driven by the number of subunits, one may set $\Psi \propto n_{0}$. If there is an optimal cluster size due to the chemical nature of the elementals, one may set $\Psi \propto k_{1}\left(n_{0}-z_{1(0)}\right)$. The result of this approach is 
a normal distribution where the constant $k_{1}$ is given by the reciprocal of the variance, $k_{1}=\sigma_{1}^{-2}=1 / \overline{\left(n_{0}-z_{1(0)}\right)^{2}}$. The formation of spherical surfactant micelles could be described in this manner. The cooperativity of micelle formation and the optimal cluster size are governed by the amphiphilic nature as well as the packing parameter of the surfactants. Here approximately normal or lognormal size distributions are often found [20-23]. Other distributions can be derived depending on the definition of $\Psi$. However, in all cases the resulting chemical potential has to add up to $R T \ln \left(z_{1(0)}\right)$ as demonstrated in Eq. (24) in order to comply with Eqs. (6) and (10).

\section{RESULTS AND EXAMPLE CALCULATIONS}

In the following, the atomistic model will be illustrated in more detail. The focus is set on systems driven by a trade-off between free energy of association/dissociation on the one hand and the free energy of mixing on the other, that yield a quasicontinuous exponential size distribution. For this case the usefulness of the atomistic hierarchical approach can be demonstrated most concisely. In the first section a qualitative examination will be given and the connections to the Schulz-Flory model of polymerization/condensation will be highlighted. Sections III B and III C deal with example calculations using experimental data. The examples are chosen to demonstrate the versatility and coherence of the approach rather than for delving into details. The approach outlined above is intended to serve as a general framework for the hierarchical stratification of a system and to obtain the free energies, enthalpies, and entropies to enhance mechanistic understanding. The examples focus on such decompositions.

\section{A. Exponential equilibrium distribution and the Schulz-Flory kinetic polymerization model}

The atomistic model defines the purely monomeric state as reference and determines the free energy difference $\Delta G_{1(0)}$ between the pure monomeric and the aggregated equilibrium state as characterized by $z_{1(0)}$. The start and end states resemble those defined by Flory's polymerization model [24]. Flory's model looks at aggregation from a kinetic perspective and considers the reaction as irreversible. Flory defines a reaction parameter $0 \leqslant p \leqslant 1$ that describes the progress of a polymerization reaction. In the context of aggregation, the reaction parameter is defined as

$$
p=\frac{\left(N_{0}^{T}-N\right)}{N_{0}^{T}} .
$$

Here $N_{0}^{T}$ is the total number of monomers/particles in the system that can take part in a condensation/aggregation reaction. $\left(N_{0}^{T}-N\right)$ is time dependent and denotes the number of particles that have reacted and are not present as monomers anymore. If we consider the degree of aggregation of these $\left(N_{0}^{T}-N\right)$ reacted particles as $n_{0}$, the fraction of monomers in $n_{0}$-mers is given by the probability distribution function

$$
P\left(n_{0}\right)=n_{0} p^{n_{0}-1}(1-p)^{2} .
$$

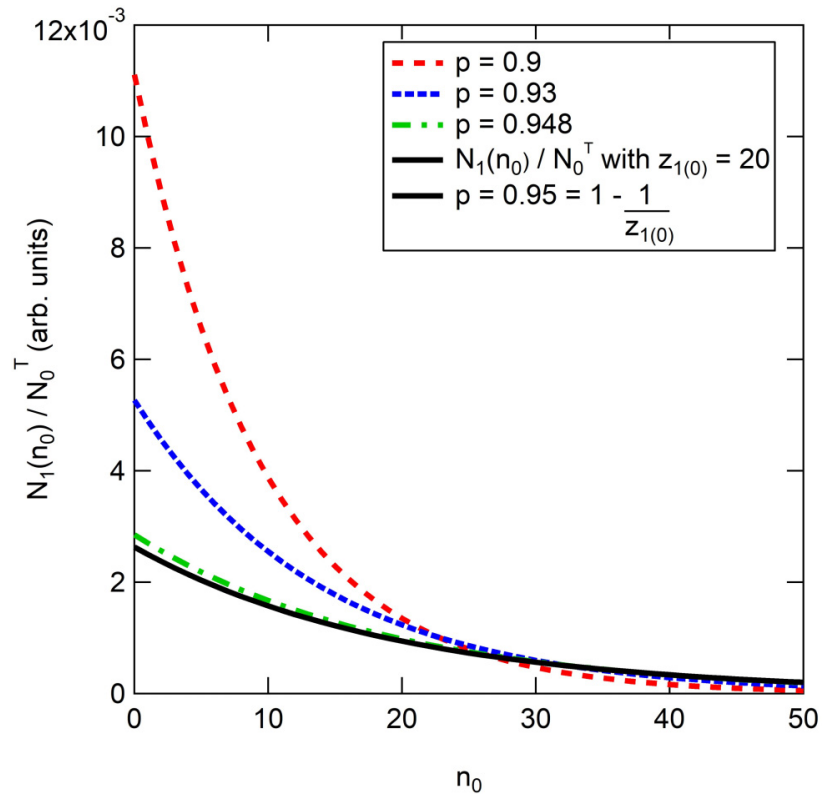

FIG. 3. For large $z_{1(0)}$ an aggregation/polymerization reaction can be considered quasi-irreversible and $1 / z_{1(0)} \approx 1-p$. The agreement between the molar Schulz-Flory distribution and $N_{1}\left(n_{0}\right)$ from the atomistic model for simple aggregation improves with increasing $z_{1(0)}$. The last two curves in the legend overlap in the plot.

The number of particles consisting of $n_{0}$ subunits/segments $N_{1}\left(n_{0}\right)$ is given by

$$
P\left(n_{0}\right)\left(\frac{N_{0}^{T}}{n_{0}}\right)=N_{1}\left(n_{0}\right)=N_{0}^{T} p^{n_{0}-1}(1-p)^{2} .
$$

The exponential form of Eq. (27) is given by

$$
N_{1}\left(n_{0}\right)=N_{0}^{T}(1-p)^{2} \exp \left\{-\left(n_{0}-1\right)(-\ln p)\right\} .
$$

Since $-\ln (p) \approx 1-p$ if $p \approx 1$,

$$
\begin{aligned}
\lim _{p \rightarrow 1} N_{1}\left(n_{0}\right) & =N_{0}^{T}(1-p)^{2} \exp \left\{-\left(n_{0}-1\right)(1-p)\right\} \\
& \approx \lim _{z_{1(0)} \rightarrow \infty}\left[\frac{N_{0}^{T}}{\left(z_{1(0)}\right)^{2}} \exp \left(\frac{-n_{0}}{z_{1(0)}}\right)\right] .
\end{aligned}
$$

The comparison with Eq. (18) demonstrates that in this limit $z_{1(0)}=1 /(1-p)$. However, because of the constraint $p \rightarrow 1$ the relation can just be true for very large values of $z_{1(0)}$, i.e., $z_{1(0)} \rightarrow \infty$. This in turn leads to a free energy difference $\Delta G_{1(0)}=R T \ln \left(z_{1(0)}\right)$ that increases without boundaries. Bearing in mind that $\Delta G_{1(0)}$ was defined as the free energy difference for the dissociation reaction (see the Theory section), this means that the free energy of association, $-\Delta G_{1(0)}$, decreases without limits. This is a characteristic of an irreversible reaction, as premised by Flory [24].

From a thermodynamic point of view there are no perfect irreversible chemical reactions in nature. The presence of thermal energy $R T$ and the finite energy of ground states implies that there is always a finite probability that a subunit or segment dissociates from an aggregate or composite particle. The premise of an irreversible reaction approximates simpler theoretical expressions. In reality a polymerization or condensation reaction that is governed by a trade-off between 


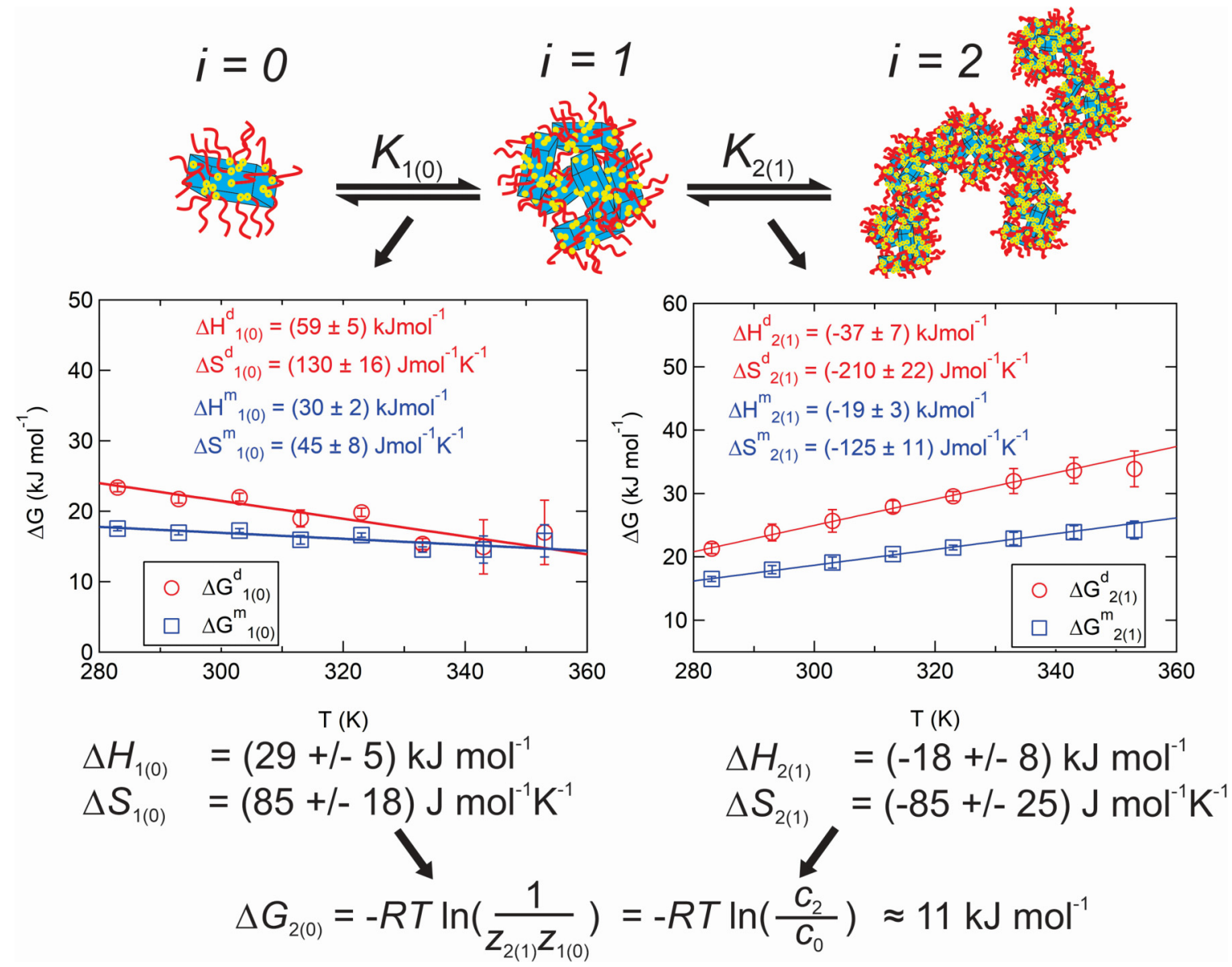

FIG. 4. Structural hierarchies for the surfactant stabilized pigment dispersion of PY14 (5.5 wt $\%$ in $\mathrm{H}_{2} \mathrm{O}$ ) yielding clusters/aggregates of first and second kind (top). From small-angle and ultra small-angle x-ray scattering (SAXS \& USAXS) measurements at different temperatures the distributions $N_{1}\left(n_{0}\right)$ and $N_{2}\left(n_{1}\right)$ and change in free energies for dissociation can be determined (middle). The results confirm that the total free energy change for dissociation is a sum of the individual contributions according to Eq. (24). The presented data were adapted from Ref. [26].

an attractive energy $\Delta G_{1(0)}^{d}$ or $\Delta G_{1(0)}^{a}$ and the mixing entropy $\Delta S_{1(0)}^{m}$ has to reach equilibrium at a finite $z_{1(0)}$ when $N_{1}\left(n_{0}\right)$ adopts an overall exponential distribution that marks the end of the net reaction at a value of $p<1$ (see Fig. 3):

$$
p=1-\frac{1}{z_{1(0)}}=1-\exp \left(\frac{-\Delta G_{1(0)}}{R T}\right) .
$$

If a given number distribution $N_{1}\left(n_{0}\right)$ exhibits an overall exponential distribution already at low values of $p$, this might be an indication that the equilibrium state is already achieved and that $\Delta G_{1(0)}$ is not large enough to drive the reaction towards large values $z_{1(0)}$. Figure 3 demonstrates that for average aggregation values $z_{1(0)}$ as low as 20 the criterion of quasi-irreversibility is approximately fulfilled. The atomistic hierarchical approach, however, predicts that the net reaction will stop at a finite $p=1-\frac{1}{\left.z_{10}\right)}$ and allows for the calculation of its free energy difference $\Delta G_{1(0)}$ with respect to the starting point of the reaction, the purely monomeric state.

\section{B. Pigment particles in aqueous dispersions}

Pigment Yellow 14 (PY14) is a common pigment used for a variety of applications including ink-jet ink. A model aqueous formulation for an ink includes PY14 nanoparticles and a nonionic surfactant, Triton $\mathrm{X}-100 \AA$. Under these conditions the pigment displays three levels of hierarchy. Elemental crystals form compact clusters that in turn assemble to less dense, fractal aggregates. This stable colloidal suspension is subject to thermal equilibrium [25].

Whether these dispersions will just form monomeric particles $i=0$, i.e., a core formed by pigment crystals surrounded by a shell of surfactants, or whether the monomers will aggregate into clusters $i>0$ is a question of the mutual interactions. A shell of charged surfactants will promote dispersion and hinder aggregation to higher assemblies. The long-range repulsive interactions do not allow short-range attractive forces to come into effect. However, short-range attractive interactions like van der Waals interactions can lead to the formation of clusters if nonionic surfactants like Triton X-100 are used which display lower solution-critical temperature phase behavior in water.

Figure 4 depicts the dissociation enthalpies and entropies for clusters $(i=1)$ and aggregates $(i=2)$ of the pigment PY14 system [26]. The equilibrium concentrations of Triton X-100 solubilized pigment particles as well as the concentrations of primary and secondary aggregates were 


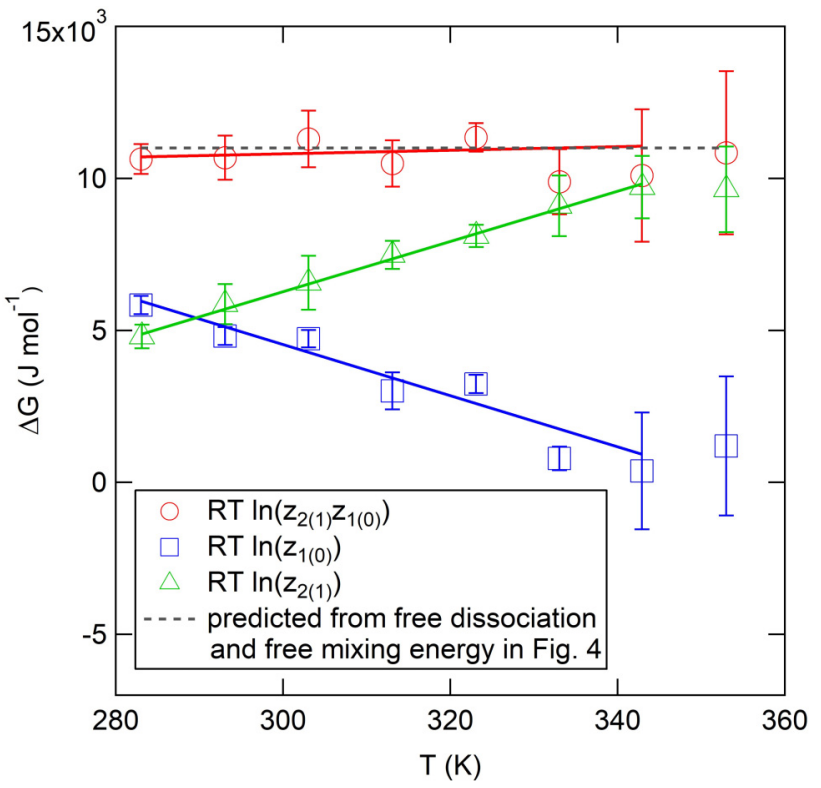

FIG. 5. Change in free energies, $\Delta G_{1(0)}, \Delta G_{2(1)}$, and $\Delta G_{2(0)}$ according to Eq. (31) for association/dissociation of pigment Pigment yellow 14 with three hierarchical levels [26]. Note that the cloud point of Triton X-100 under these conditions $(0.1 \mathrm{wt} \%)$ is about $340 \mathrm{~K}$ [30]. The data points at $353 \mathrm{~K}$ were therefore excluded from the fits.

determined with small-angle x-ray scattering at different temperatures. From these values the free dissociation energies $R T \ln \left[\frac{\left(z_{1(0)}\right)^{2}}{\phi}\right]$ and free mixing energies $R T \ln \left(\frac{N_{1}^{T}}{N_{\Omega .0}}\right)$ were determined. Note that the error bars depict the largest possible inaccuracy calculated by error propagation. The final value for $\Delta G_{1(0)}$ is therefore shown to the second significant digit for a better comparison with the calculation. From the balance between the free dissociation energies and free mixing energies one would conclude that the overall free energy change on dissociation amounts to about $11 \mathrm{~kJ} \mathrm{~mol}^{-1}$. The entropic terms nearly cancel each other.

On the other hand, employing Eq. (6) and formulating the osmotic pressure of a system with three hierarchical levels $(i=0,1,2)$, one obtains

$$
\begin{aligned}
\ln \left(\frac{\Pi_{2}}{c_{0} R T}\right) & =\ln \left(\frac{c_{2}}{c_{0}}\right)=\ln \left(\frac{c_{2}}{c_{1}} \frac{c_{1}}{c_{0}}\right)=\ln \left(K_{2(1)} K_{1(0)}\right) \\
& =-\ln \left(z_{2(1)} z_{1(0)}\right)=\frac{-1}{R T}\left(\Delta G_{2(1)}+\Delta G_{1(0)}\right) \\
& =\frac{-\Delta G_{2(0)}}{R T}
\end{aligned}
$$

where $\Pi_{2}$ is the overall osmotic pressure and $c_{2}$ the overall concentration of particles in the aggregated system (elementals as well as primary and secondary aggregates).

Figure 5 shows a plot of $R T \ln \left(z_{1(0)}\right)$ and $R T \ln \left(z_{2(1)}\right)$ versus temperature $T$, which should yield lines that correspond to the values found in Fig. 4 and sum up to $\Delta G_{2(0)}$. Figure 5 demonstrates that this is indeed the case. By linear regression, values of (30 \pm 2$),(-19 \pm 3)$, and $(9 \pm 4) \mathrm{kJ} \mathrm{mol}^{-1}$ are found for $\Delta H_{1(0)}, \Delta H_{2(1)}$, and $\Delta H_{2(0)}$, respectively. The entropies $\Delta S_{1(0)}, \Delta S_{2(1)}$, and $\Delta S_{2(0)}$ amount to $(84 \pm 8)$,
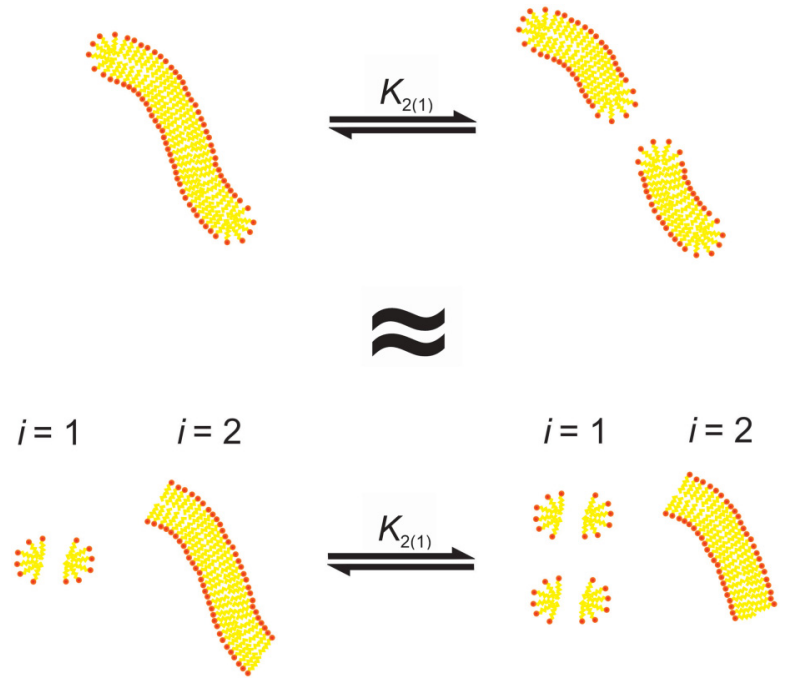

FIG. 6. Structural hierarchies for WLMs. The surfactants can either reside in a hemispherical end cap or in the denser cylindrical geometry connecting the end caps. The two states are energetically not equivalent. Scission leads to an increase of the surfactant population residing in end caps. The smallest entity that can be created by scission consists of two hemispherical end caps, i.e., a spherical micelle.

$(-83 \pm 11)$, and $(6 \pm 14) \mathrm{J} \mathrm{mol}^{-1} \mathrm{~K}^{-1}$, respectively. Within the accuracy the balance performed in Fig. 4 matches with the ones obtained by relation (31). The latter values, however, are more accurate since fewer variables are employed for calculation. The x-ray scattering measurements in Ref. [26] were analyzed using the structural-hierarchical model of Beaucage [27-29], underlining the congruency of the structural and the thermodynamic approach.

With the atomistic hierarchical approach a much subtler decomposition of the forces governing cluster formation of the pigment is possible. The analysis reveals and quantifies that the number of subunits for the primary clusters $i=1$ decreases with increasing temperature. For the secondary aggregates $i=2$ the opposite trend is observed. For pigment systems, this information may be invaluable to understand the temperature dependent optical properties like the saturation, haze, and luster. After separating the driving forces as outlined above, the free energies in turn can be employed to deepen the understanding of the aggregate structures, e.g., fractal dimension or branching.

\section{Free energy of scission of wormlike micelles}

The final example deals with a less obvious case of structural hierarchy and demonstrates that the explicit assignment of the hierarchical levels can deepen the understanding of supposedly easy systems. Wormlike micelles (WLMs) are structures assembled from surfactant molecules in an aqueous environment. While at low surfactant and salt concentrations spherical micelles $(i=1)$ are found, addition of further surfactant or salt leads to the assembly of elongated, threadlike structures $(i=2)$ (see Fig. 6) [31]. Below the overlap concentration the structure of wormlike micelles can be determined using small-angle neutron scattering. When the length, 


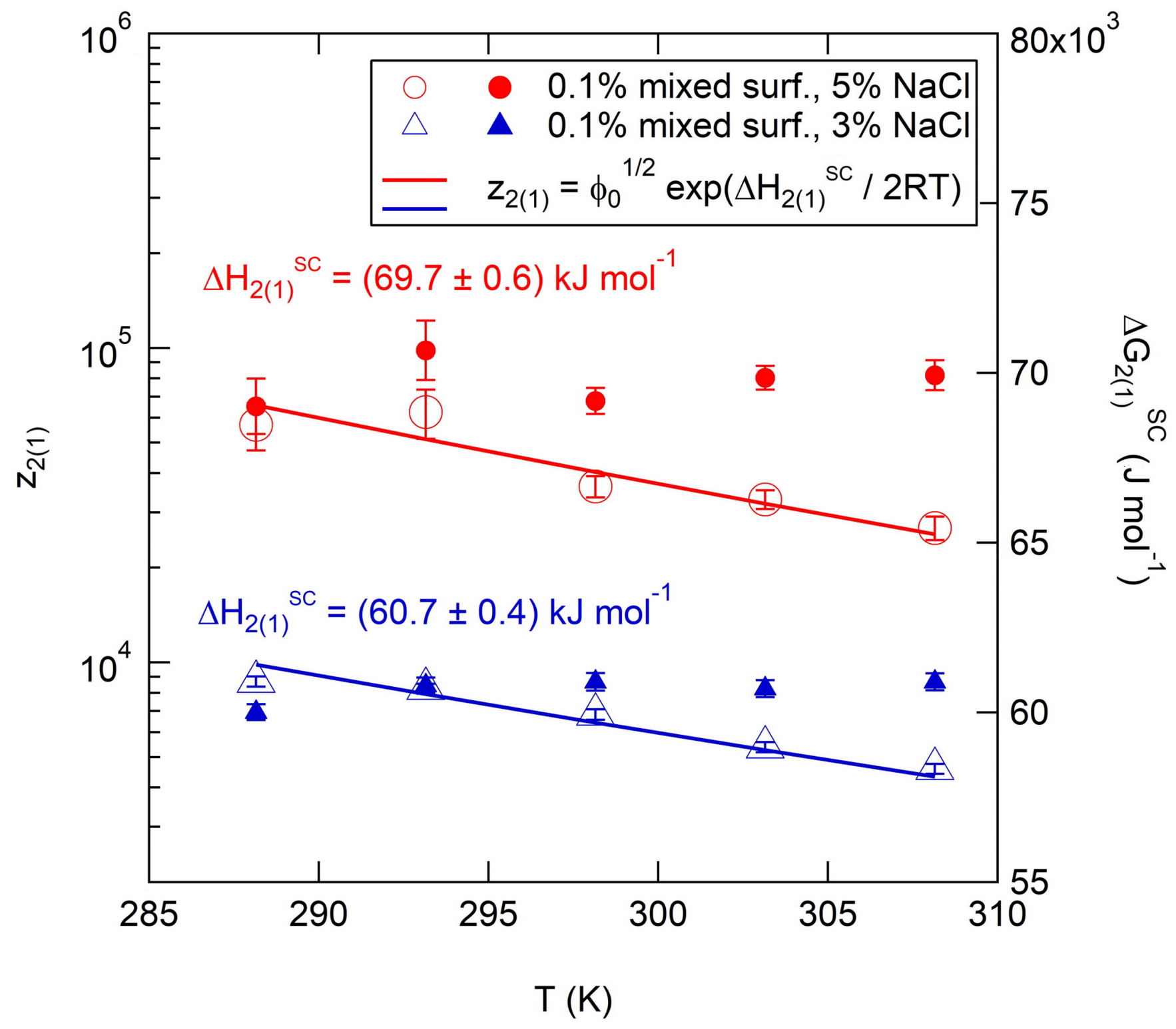

FIG. 7. Semilogarithmic plot of average aggregation number $z_{2(1)}$ (read from the left axis) versus temperature for a mixed surfactant system (sodium laureth-1 sulfate and cocoamidopropyl betaine 9:1) in $\mathrm{D}_{2} \mathrm{O}$ with 5 and $3 \mathrm{wt} \% \mathrm{NaCl}$ denoted by open red circles and open blue triangles respectively; the free scission energies $\Delta G_{2(1)}^{s c}$ (read from the right axis) as a function of temperature for the mixed surfactant system with 5 and $3 \mathrm{wt} \% \mathrm{NaCl}$ denoted by solid red circles and solid blue triangles, respectively. For both cases, $\Delta G_{2(1)}^{s c}$ is nearly independent of temperature.

diameter, and density of these threadlike structures are determined, the average aggregation number $z_{2(1)}$ can be calculated and employed together with the volume fraction $\phi$ to determine the free energy of scission $\Delta G_{2(1)}^{S C}$ [32]. The fit parameters to small-angle neutron scattering are listed in the Appendix.

Figure 7 shows the resulting average aggregation number $z_{2(1)}$ as function of temperature for two salt concentrations ( 3 and $5 \mathrm{wt} \% \mathrm{NaCl}$ ). At higher salt concentration the WLMs are much larger, in agreement with literature data [31]. The wormlike micelles break and merge, and in the process two end caps are formed or removed, respectively (see Fig. 6). The free energy of surfactant molecules residing in the cylindrical part of the WLM is not the same as for surfactants located in an end cap. Since every WLM exhibits just two end caps, the size of the individual WLM does not play a role for the free energy of scission, but only affects the mixing entropy. In terms of structural hierarchies, the system consists of surfactants in hemispherical end caps on the one hand and surfactants residing in the elongated, cylindrical geometry on the other.

The presence of ionic compounds like salt or salicylate screens Coulombic interactions, so that a cylindrical packing geometry with smaller head-group/head-group distances becomes more favorable, leading to an elongation of the WLMs [31,33,34]. While the repulsion between the charged, hydrophilic head groups disfavors the cylindrical geometry, the hydrophobic effect favors a dense packing of the surfactant molecules with smaller head-group/head-group distances. Here the hydrophobic tails of the surfactant molecules are 
shielded much better from contact with water molecules; i.e., the solvent accessible surface area is smaller. The mixing entropy $\Delta S_{2(1)}^{m}$, however, fosters a large dispersion of small particles with a large overall number of end caps.

Within the accuracy of the experiment the free energy $\Delta G_{2(1)}^{S C}$ is roughly constant in temperature (read from right axis in Fig. 7), so that the depicted scission free energy can be identified with an enthalpy. Since the hydrophobic effect is involved, a thermodynamic description of the process employing the change of heat capacity $\Delta C_{P}$ would probably be more appropriate [35]. The error bars and the limit of the employed method when the aggregates become very large (see data at $5 \mathrm{wt} \% \mathrm{NaCl}$ and low temperatures) do not allow for this kind of analysis. However, for the given experimental conditions and accuracy the results underline that it is indeed the thermodynamic pseudopotential expressed in relation (21) that governs the assembly.

\section{CONCLUSIONS}

While most aggregates are obtained from kinetically limited growth processes, some self-assembled and equilibrated aggregates exist and there are some cases where a pseudoequilibrium model can be of use. For these aggregates a thermodynamic analysis is possible and is useful in controlling growth. An atomistic hierarchical theory is presented that couples well with small-angle scattering, simulation, and other analytic techniques that can quantify the average degree of aggregation which is directly used to calculate the free energy, enthalpy, and entropy change on aggregation.

The new approach is demonstrated for three systems, condensation polymerization, surfactant stabilized pigments, and wormlike micelles.

\section{ACKNOWLEDGMENTS}

The partial funding of this work through the Procter \& Gamble Company is gratefully acknowledged. The authors would like to thank Michael Weaver, P\&G Analytical Sciences, for his support and assistance regarding wormlikemicellar systems. A portion of this research used resources at the High Flux Isotope Reactor, a DOE Office of Science User Facility operated by the Oak Ridge National Laboratory. G.B., A.M., and K.R. were supported by the National Science Foundation through Grant No. CMMI-1635865. X-ray scattering data for the pigment samples were collected at the Advanced Photon Source (APS) on beam line 9-ID-C operated by the X-ray Science Division. Use of the APS, an Office of Science User Facility operated for the U.S. Department of Energy (DOE) Office of Science by Argonne National Laboratory, was supported by the U.S. DOE under Contract No. DEAC02-06CH11357.

\section{APPENDIX: MATERIALS, METHODS AND SANS RESULTS FOR WLMS}

Sample material, preparation, and measurements for the wormlike micelles were similar to those described by Vogtt et al. [36]. The small-angle neutron measurements were conducted at the Bio-SANS instrument at the Oak Ridge

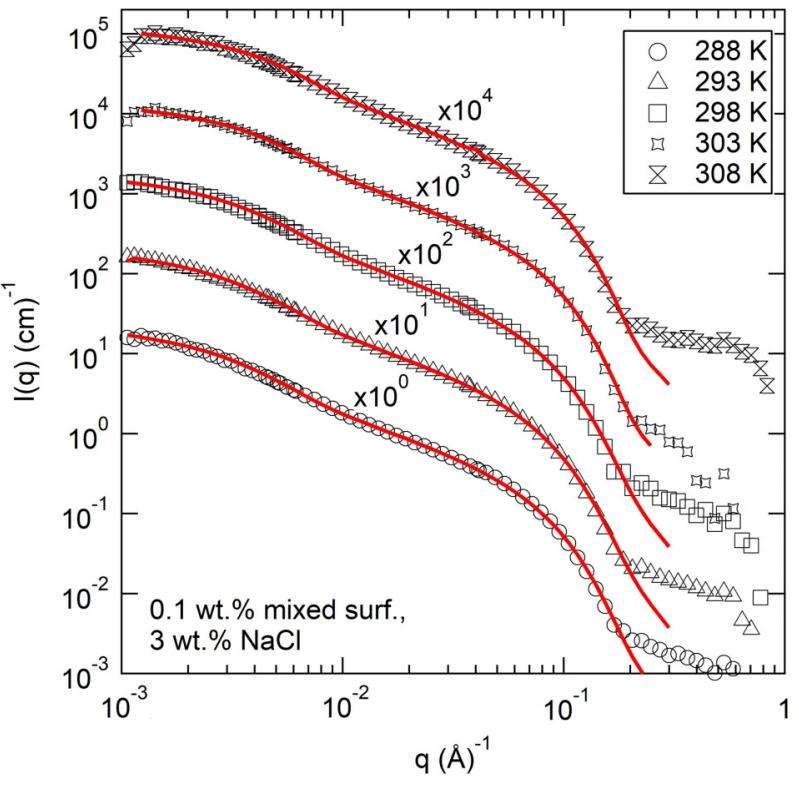

FIG. 8. Scattered intensity $I(q)$ versus momentum transfer $q$ for $0.1 \%$ mixed surfactant (sodium laureth-1 sulfate and cocoamidopropyl betaine $9: 1)$ in $\mathrm{D}_{2} \mathrm{O}(3 \% \mathrm{NaCl})$ at various temperatures. The red lines depict fits according to a wormlike-chain model yielding the length $L_{1}$ and radius $R_{1}$ of the cylindrical segments as well as the number of segments, $z_{2(1)}$ (see Table I) [32].

National Laboratory in Oak Ridge, TN. Data analysis was performed as described by Vogtt et al. [37], except that the scattering fit parameters $G_{2}$ and $R_{g, 2}$ were substituted for

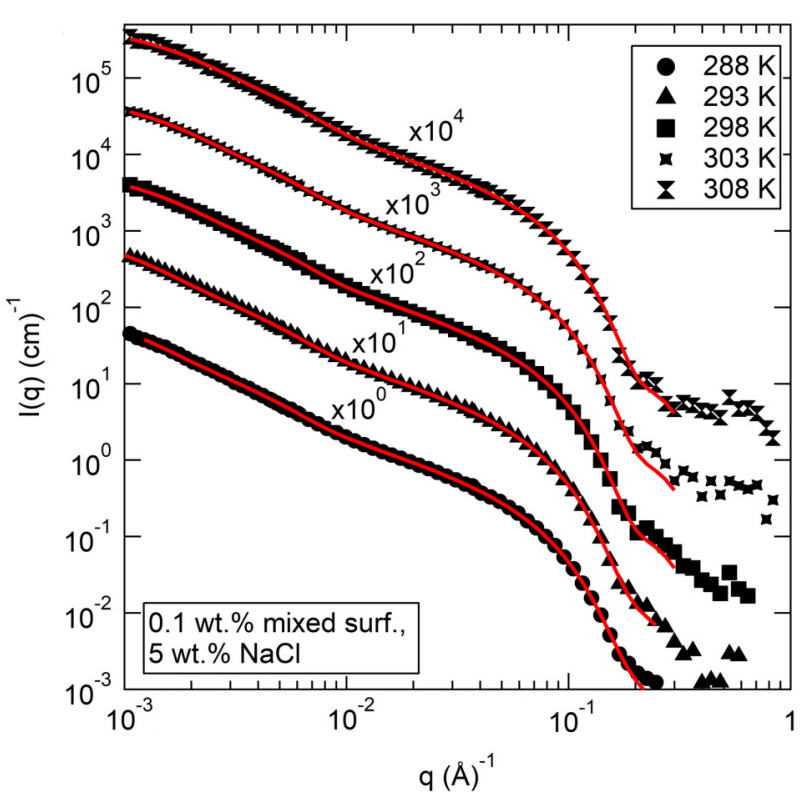

FIG. 9. Scattered intensity $I(q)$ versus momentum transfer $q$ for $0.1 \%$ mixed surfactant (sodium laureth-1 sulfate and cocoamidopropyl betaine 9:1) in $\mathrm{D}_{2} \mathrm{O}(5 \% \mathrm{NaCl})$ at various temperatures. The red lines depict fits according to a wormlike-chain model. The fitting results are given in Table I. The values allow calculating the average aggregation number $z_{2(1)}$ and the Gibbs free energy of scission, $\Delta G_{2(1)}^{s c}$, shown in Fig. 7. 
the number of cylindrical subunits $z_{2(1)}$ using the following relations $[37,38]$ :

$$
\begin{gathered}
G_{2}=\left(z_{2(1)}-1\right) G_{1}, \\
R_{g, 2} \approx\left\{\frac{1.75\left(z_{2(1)} \frac{2}{d_{f, 2}}\right) L_{1}^{2}}{\frac{\left(d_{f, 2}+2\right)}{d_{\min , 2}} \frac{\left(d_{\min , 2}+d_{f, 2}+2\right)}{d_{\min , 2}}}\right\}^{\frac{1}{2}} .
\end{gathered}
$$

Figures 8 and 9 show the scattered intensity $I(q)$ as a function of momentum transfer $q$ for $0.1 \%$ mixed surfactant (9:1 sodium laureth-1-sulfate:cocoamidopropyl betaine) in deuterated water with $3 \%$ and $5 \% \mathrm{NaCl}$ at various temperatures, respectively. The solid red lines in both figures depict fits according to a wormlike-chain model described by Vogtt et al. [37]. The fit parameters for structural level $i=1$, length $L_{1}$, and radius $R_{1}$ and $\sigma_{R 1}$ of the cylindrical segments as well as the number of segments $z_{2(1)}$ are listed in Table I. Additionally, the parameters $d_{f, 2}$ and $d_{\min , 2}$ were set to the value 1.67 that corresponds to a self-avoiding walk of the segments in the wormlike chain rendering the remaining fit parameters in Eqs. (A1) and (A2) solely a function of $z_{2(1)}$. The average aggregation number $z_{2(1)}$ was used to determine the Gibbs free energy of scission, $\Delta G_{2(1)}^{s c}$ shown in Fig. 7.

TABLE I. Fitting parameters for $0.1 \mathrm{wt} \%$ mixed surfactant in $\mathrm{D}_{2} \mathrm{O}(3$ and $5 \mathrm{wt} \% \mathrm{NaCl})$ at various temperatures. The parameters $d_{f, 2}$ and

\begin{tabular}{|c|c|c|c|c|c|}
\hline Temperature & $\begin{array}{c}\phi(\Delta \rho)^{2} \\
\left(10^{22} \mathrm{~cm}^{-4}\right)\end{array}$ & $\begin{array}{l}R_{1} \\
(\AA)\end{array}$ & $\sigma_{R 1}$ & $\begin{array}{l}L_{1} \\
(\AA)\end{array}$ & $z_{2(1)}$ \\
\hline \multicolumn{6}{|c|}{$3 \% \mathrm{NaCl}$} \\
\hline $288 \mathrm{~K}$ & $4.58 \pm 0.03$ & $16.3 \pm 0.2$ & $0.264 \pm 0.006$ & $882 \pm 6$ & $5.6 \pm 0.1$ \\
\hline $293 \mathrm{~K}$ & $4.37 \pm 0.03$ & $16.0 \pm 0.2$ & $0.285 \pm 0.006$ & $854 \pm 6$ & $5.7 \pm 0.1$ \\
\hline $298 \mathrm{~K}$ & $4.42 \pm 0.03$ & $15.8 \pm 0.2$ & $0.279 \pm 0.006$ & $817 \pm 5$ & $5.0 \pm 0.1$ \\
\hline $303 \mathrm{~K}$ & $4.45 \pm 0.03$ & $16.4 \pm 0.2$ & $0.234 \pm 0.007$ & $796 \pm 5$ & $3.79 \pm 0.07$ \\
\hline $308 \mathrm{~K}$ & $4.53 \pm 0.03$ & $16.0 \pm 0.2$ & $0.242 \pm 0.007$ & $753 \pm 5$ & $3.61 \pm 0.06$ \\
\hline \multicolumn{6}{|c|}{$5 \% \mathrm{NaCl}$} \\
\hline $288 \mathrm{~K}$ & $4.25 \pm 0.02$ & $18.5 \pm 0.2$ & $0.243 \pm 0.005$ & $900 \pm 5$ & $28 \pm 4$ \\
\hline $293 \mathrm{~K}$ & $4.20 \pm 0.02$ & $18.6 \pm 0.2$ & $0.227 \pm 0.005$ & $869 \pm 5$ & $33 \pm 5$ \\
\hline $298 \mathrm{~K}$ & $4.36 \pm 0.02$ & $18.3 \pm 0.2$ & $0.209 \pm 0.006$ & $819 \pm 5$ & $20 \pm 1$ \\
\hline $303 \mathrm{~K}$ & $4.41 \pm 0.03$ & $17.8 \pm 0.2$ & $0.210 \pm 0.006$ & $788 \pm 5$ & $20 \pm 1$ \\
\hline $308 \mathrm{~K}$ & $4.43 \pm 0.03$ & $17.7 \pm 0.2$ & $0.206 \pm 0.006$ & $750 \pm 4$ & $17.2 \pm 0.8$ \\
\hline
\end{tabular}
$d_{\min , 2}$ were set to the value 1.67 corresponding to a self-avoiding walk of the segments in the wormlike chain.

[1] M. v. Smoluchowski, Z. Phys. Chem. 92U, 129 (1918).

[2] T. A. Witten and L. M. Sander, Phys. Rev. Lett. 47, 1400 (1981).

[3] M. Y. Lin, H. M. Lindsay, D. A. Weitz, R. C. Ball, R. Klein, and P. Meakin, Nature 339, 360 (1989).

[4] S. K. Friedlander, Smoke, Dust, and Haze: Fundamentals of Aerosol Dynamics, 2nd ed. (Oxford University Press, New York, 2000).

[5] T. Sugimoto, Monodispersed Particles, 2nd ed. (Elsevier, Amsterdam, 2019).

[6] Y. Jin, G. Beaucage, K. Vogtt, H. Jiang, V. Kuppa, J. Kim, J. Ilavsky, M. Rackaitis, A. Mulderig, K. Rishi, and V. Narayanan, Polymer (Guildf.) 129, 32 (2017).

[7] A. Mulderig, G. Beaucage, K. Vogtt, H. Jiang, Y. Jin, L. Clapp, and D. C. Henderson, Langmuir 33, 14029 (2017).

[8] K. Rishi, V. Narayanan, G. Beaucage, A. McGlasson, V. Kuppa, J. Ilavsky, and M. Rackaitis, Polymer (Guildf.) 175, 272 (2019).

[9] G. Odriozola, A. Moncho-Jordá, A. Schmitt, J. CallejasFernández, R. Martínez-García, and R. Hidalgo-Álvarez, Europhys. Lett. 53, 797 (2001).

[10] G. Odriozola, A. Schmitt, A. Moncho-Jordá, J. CallejasFernández, R. Martínez-García, R. Leone, and R. HidalgoÁlvarez, Phys. Rev. E 65, 031405 (2002).

[11] A. M. Puertas and G. Odriozola, J. Phys. Chem. B 111, 5564 (2007).
[12] M. Thorn and M. Seesselberg, Phys. Rev. Lett. 72, 3622 (1994).

[13] M. Thorn, M. L. Broide, and M. Seesselberg, Phys. Rev. E 51, 4089 (1995).

[14] A. I. Rusanov, Adv. Colloid Interface Sci. 45, 1 (1993).

[15] A. I. Rusanov, Adv. Colloid Interface Sci. 38, 271 (1992).

[16] A. Ben-Naim, A Farewell to Entropy: Statistical Thermodynamics Based on Information (World Scientific, Singapore, 2008).

[17] M. E. Cates, J. Phys. 49, 1593 (1988).

[18] M. E. Cates and S. J. Candau, J. Phys.: Condens. Matter 2, 6869 (1990).

[19] M. E. Cates and S. M. Fielding, Adv. Phys. 55, 799 (2006).

[20] C. M. Beliciu and C. I. Moraru, J. Dairy Sci. 92, 1829 (2009).

[21] N. Yoshii, K. Iwahashi, and S. Okazaki, J. Chem. Phys. 124, 184901 (2006).

[22] J. N. Israelachvil, D. J. Mitchell, B. W. Ninham, J. N. Israelachvili, D. J. Mitchell, and B. W. Ninham, J. Chem. Soc. Faraday Trans. 2 72, 1525 (1976).

[23] C. Tanford, Proc. Natl. Acad. Sci. USA 71, 1811 (1974).

[24] P. J. Flory, J. Am. Chem. Soc. 58, 1877 (1936).

[25] Y. Rharbi, M. Li, M. A. Winnik, and K. G. Hahn, J. Am. Chem. Soc. 122, 6242 (2000).

[26] K. Rishi, A. Mulderig, G. Beaucage, K. Vogtt, and H. Jiang, Langmuir 35, 13100 (2019). 
[27] G. Beaucage and D. W. W. Schaefer, J. Non.-Cryst. Solids 172174, 797 (1994).

[28] G. Beaucage, J. Appl. Cryst. 28, 717 (1995).

[29] G. Beaucage, J. Appl. Cryst. 29, 134 (1996).

[30] L. Koshy, A. H. Saiyad, and A. K. Rakshit, Colloid Polym. Sci. 274, 582 (1996).

[31] C. A. Dreiss, Soft Matter 3, 956 (2007).

[32] K. Vogtt, H. Jiang, G. Beaucage, and M. Weaver, Langmuir 33, 1872 (2017).

[33] T. S. Davies, A. M. Ketner, and S. R. Raghavan, J. Am. Chem. Soc. 128, 6669 (2006).
[34] Z. Lin, J. J. Cai, L. E. Scriven, and H. T. Davis, J. Phys. Chem. 98, 5984 (1994).

[35] W. Blokzijl and J. B. F. N. Engberts, Angew. Chem. Int. Ed. Engl. 32, 1545 (1993).

[36] K. Vogtt, G. Beaucage, M. Weaver, and H. Jiang, Soft Matter 13, 6068 (2017).

[37] K. Vogtt, G. Beaucage, M. Weaver, and H. Jiang, Langmuir 31, 8228 (2015).

[38] R. Ramachandran, G. Beaucage, A. S. Kulkarni, D. McFaddin, J. Merrick-Mack, and V. Galiatsatos, Macromolecules 41, 9802 (2008). 